\title{
Dynamic-Double-Threshold Energy Detection Scheme under Noise Varying Environment in Cognitive Radio System
}

\author{
Sonam Shrivastava \\ Dept. of Electrical Engg. \\ National Institute of Technology \\ Rourkela, India
}

\author{
Ravi Tiwari \\ Dept. of Electrical Engg. \\ National Institute of Technology \\ Rourkela, India
}

\author{
Susmita Das \\ Dept. of Electrical Engg. \\ National Institute of Technology \\ Rourkela, India
}

\begin{abstract}
In cognitive radio, spectrum sensing is a key component for securing the licensed terminal from interference and detects the white spectrum hole to improve the spectrum efficiency. In the existing techniques, the noise uncertainty was either not considered or only detrimental effects are mitigated without much performance improvement. Therefore, a novel dynamicdouble-threshold energy detection scheme is proposed under noise uncertainty, and its performance has been studied. Simulation analysis and results show that the proposed scheme improves the performance of detection for smaller values of false alarm probability. It is also found that the detection probability is reached at a satisfactory level, even under varying noise uncertainty.
\end{abstract}

\section{General Terms}

Cognitive radio, energy detection technique, spectrum sensing.

\section{Keywords}

Cognitive radio, dynamic-double-threshold, noise uncertainty, white spectrum hole, noise variance, energy detection.

\section{INTRODUCTION}

Nowadays, there is a scarcity of the radio spectrum due to advancement in wireless appliances and services as Wi-Fi, Bluetooth, ZigBee, and Wi-MAX etc. Because of this huge demand of radio spectrum cognitive radio gains much attention as it can sense the unused spectrum i.e. spectrum holes under license band and keeping spectrum utilization and quality of service high for the overall system. To meet the excessive demand of the radio spectrum band the cognitive radio $(\mathrm{CR})$ is a very promising technique as it does not cause harmful interference to the licensed terminals.

According to a survey performed by Spectrum Policy Task Force (SPTF) within the Federal Communication Commission (FCC), that actual licensed spectrum is inefficiently utilized [1-3]. To utilize the available spectrum up to the full extent it is mandatory to allow the unlicensed terminals to borrow unused licensed band under the condition that it should not cause any harm to the licensed terminals. For this, an intelligent wireless communication system is required, which must be aware of its environment and able to select the spectrum band as well as the parameter (such as carrier frequency, modulation type, Bandwidth, etc.). The cognitive radio is capable of doing this and thus fills the unused licensed spectrum holes also called as white spectrum holes, without causing much interference to the licensed terminals
[4]. In order to do so CR must sense the spectrum continuously. The basic spectrum sensing techniques, which already have been proposed, are energy detection technique [5], matched filter detection technique and cyclostationary feature detection technique [6]. In these basic transmitter detection techniques, energy detection is generally used because of its low complexity. The double threshold technique is introduced in [7]. Most of the techniques presented on the basis of invariant noise power, whereas in total noise is present due to various factors like quantization, leakage of signals, thermal noise, etc., thus we can say noise is not perfectly Gaussian also not stationary [8]. Thus, under practical scenario we cannot fix the noise variance of received signal which, results in the noise uncertainty condition.

The problem of noise uncertainty is corrected by using the proposed dynamic-double-threshold scheme. Here two varying thresholds has been taken, which will result in high detection probability, while keeping false alarm probability at fixed lower value as in the case of fixed noise condition.

The remaining part of the paper is organized as follows. In section II detection model is explained. Double threshold is introduced in section III, and noise uncertainty with dynamic threshold is explained in section IV. The performance improvement is proven with the simulation result in section V. Finally, conclusions are drawn in section VI.

\section{DETECTION MODEL}

Fig. 1 shows the system model for spectrum sensing in cognitive radio $(\mathrm{CR})$. There are two cognitive terminals (CT): $C T_{1}$ and $C T_{2}$, one licensed terminal and one licensed terminal access point. The CTs will sense the spectrum and find the spectrum holes to maximize the radio spectrum utilization.

For detection of an unused licensed band of the radio spectrum, the basic transmitter detection techniques are used generally. These techniques can be modeled as a binary hypothesis [9] as follows:

$$
\begin{cases}H_{0}: X(n)=W(n) & , n=1,2, \ldots ., N \\ H_{1}: X(n)=h \cdot S(n)+W(n) & , n=1,2, \ldots, N\end{cases}
$$




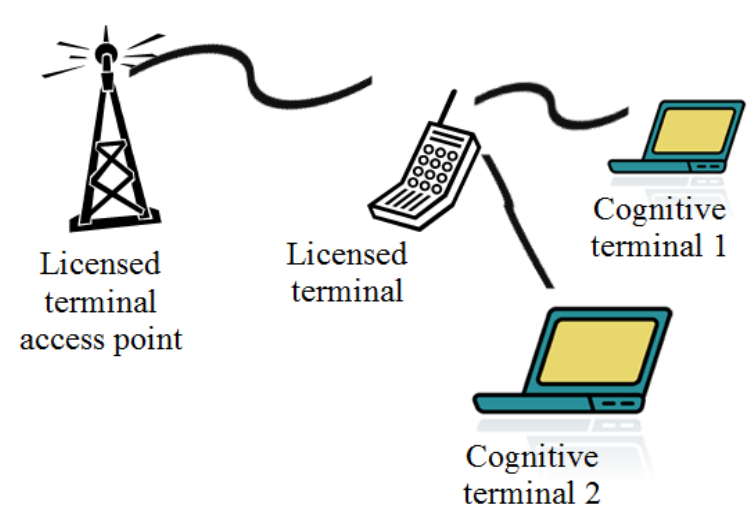

Figure 1. System model for spectrum sensing.

where, $X(n), S(n), W(n)$ and $h$ denotes the received signal at cognitive radio terminal, transmitted signal from licensed terminal, additive white Gaussian noise with zero mean and $\sigma_{n}{ }^{2}$ variance and complex channel gain respectively. $H_{0}$ represents absence of licensed terminal means spectrum band can be used by cognitive radio terminal and $H_{1}$ represents the presence of licensed terminal, i.e. spectrum band is occupied by licensed terminals [9]. Also we denote the signal to noise ratio (SNR) by $\gamma=\frac{P}{\sigma_{n}{ }^{2}}$. Where $P$ is the average signal power and $\sigma_{n}{ }^{2}$ is noise variance. The following cases can be drawn from the binary hypothesis:

1) Probability of detection $\left(\boldsymbol{P}_{\boldsymbol{d}}\right)$ : The Channel is vacant and declared as vacant.

2) Probability of false alarm $\left(\boldsymbol{P}_{\boldsymbol{f}}\right)$ :The Channel is vacent and declared as occupied.

3) Probability of mis detection $\left(\boldsymbol{P}_{\boldsymbol{m}}\right)$ :The Channel is occupied and declared as vacant.

The test statistics for energy detection method is given as [10],

$$
D(X)=\frac{1}{N} \sum_{n=0}^{N-1}[X(n)]^{2}
$$

Here, $D(X)$ is test statics, $N$ is the number of samples taken under observation period. For a single threshold value $\lambda$, presence or absence of licensed terminal can be declared as:

$$
\left\{\begin{array}{c}
D(X)>\lambda, H_{1} \text { : licensed terminal is present } \\
D(X)<\lambda, H_{0} \text { :licensed terminal is absent }
\end{array}\right.
$$

If we assume that noise variance is fixed and noise uncertainty is not considered [10],

$$
D(X) \sim\left\{\begin{array}{lr}
\mathcal{N}\left(\sigma_{n}{ }^{2}, \sigma_{n}{ }^{2} / N\right) & H_{0} \\
\mathcal{N}\left(\left(\sigma_{n}{ }^{2}+P\right),\left(\sigma_{n}{ }^{2}+P\right)^{2} / N\right) & H_{1}
\end{array}\right.
$$

Now, we can derive probability of detection $\left(P_{d}\right)$, probability of false alarm $\left(P_{f}\right)$, and probability of mis-detection $\left(P_{m}\right)$ as:

$$
P_{d}=\operatorname{Pr}\left(D(X)>\lambda / H_{1}\right)=Q\left(\frac{\lambda-\left(P+\sigma_{n}{ }^{2}\right)}{\sqrt{2 / N} \cdot\left(P+\sigma_{n}{ }^{2}\right)}\right)
$$

$$
\begin{gathered}
P_{f}=\operatorname{Pr}\left(D(X)>\lambda / H_{0}\right)=Q\left(\frac{\lambda-\sigma_{n}^{2}}{\sqrt{2 / N} \cdot \sigma_{n}^{2}}\right) \\
P_{m}=1-P_{d}=1-Q\left(\frac{\lambda-\left(P+\sigma_{n}^{2}\right)}{\sqrt{2 / N} \cdot\left(P+\sigma_{n}^{2}\right)}\right)
\end{gathered}
$$

Here $Q($.$) represents the standard Gaussian complementary$ cumulative distribution function (CDF) and $\lambda$ represent is the preset threshold value.

\section{DOUBLE THRESHOLD SCHEME}

In order to improve the reliability of decision double threshold scheme is implemented. The two thresholds $\lambda_{1}$ and $\lambda_{2}$ are illustrated in Fig. 2. Also we define:

$$
\lambda_{2}-\lambda_{1}=\Delta \lambda
$$

Here, $\lambda_{1}=$ lower threshold bound,

$$
\lambda_{2}=\text { upper threshold bound. }
$$

Considering this criterion the cognitive terminal with test statics $D(X)$ value between $\lambda_{1}$ and $\lambda_{2}$ will not be considered and sensing will be performed again. When value of $D(X)$ is greater than the threshold value $\lambda_{2}$ hypothesis $H_{1}$ will be declared and when it is less than the threshold value $\lambda_{1}$ hypothesis $H_{0}$ will be declared. The probability equation will be modified as follows:

$$
\begin{gathered}
P_{d}=\operatorname{Pr}\left(D(X)>\lambda_{2} / H_{1}\right)=Q\left(\frac{\lambda_{2}-\left(P+\sigma_{n}^{2}\right)}{\sqrt{2 / N}\left(P+\sigma_{n}^{2}\right)}\right) \\
P_{f}=\operatorname{Pr}\left(D(X)>\lambda_{1} / H_{0}\right)=Q\left(\frac{\lambda_{1}-\sigma_{n}^{2}}{\sqrt{2 / N} \cdot \sigma_{n}^{2}}\right) \\
P_{m}=1-P_{d}=1-Q\left(\frac{\lambda_{2}-\left(P+\sigma_{n}^{2}\right)}{\sqrt{2 / N}\left(P+\sigma_{n}^{2}\right)}\right)
\end{gathered}
$$

The performance of this scheme will depend upon value of $\Delta \lambda$. In case if the higher value of $\Delta \lambda$ is considered then the probability that the test statistics falls in null region will be more, resulting in more number of sensing cycles for selection of white spectrum hole, and if lower value will be taken, it will result in either miss detection or false alarm. Thus Proper value of $\Delta \lambda$ should be chosen.

\section{PROBLEM FORMULATION FOR NOISE UNCERTAINTY}

The detection performance has been discussed without considering noise uncertainty in to account. Whereas, the noise variance is not fixed as the wireless channel is continuously changing.

\subsection{Impact of Noise Uncertainty}

Considering a case of varying noise variance as $\sigma^{2} \epsilon\left[{\sigma_{n}}^{2} / \rho, \rho \sigma_{n}{ }^{2}\right]$ [11] [12], where $\rho$ represents the noise uncertainty coefficient and value of $\rho$ is close to 1, i.e. $\rho>$ 1 and $\rho \approx 1$. So (9) and (10)will be modified as: 


$$
\begin{aligned}
& P_{d}=\min _{\sigma^{2} \epsilon\left[\sigma_{n}{ }^{2} / \rho, \rho{\sigma_{n}}^{2}\right]} Q\left(\frac{\lambda_{2}-\left(P+\sigma^{2}\right)}{\sqrt{2 / N} \cdot\left(P+\sigma^{2}\right)}\right) \\
& P_{d}=Q\left(\frac{\lambda_{2}-\left(P+\sigma_{n}^{2} / \rho\right)}{\sqrt{2 / N} \cdot\left(P+\sigma_{n}^{2} / \rho\right)}\right) \\
& P_{f}=\underset{\sigma^{2} \in\left[\sigma_{n}^{2} / \rho, \rho \sigma_{n}{ }^{2}\right]}{\max } Q\left(\frac{\lambda_{1}-\sigma^{2}}{\sqrt{2 / N} \cdot \sigma^{2}}\right) \\
& P_{f}=Q\left(\frac{\lambda_{1}-\rho \sigma_{n}^{2}}{\sqrt{2 / N} \cdot \rho \sigma_{n}^{2}}\right) \\
& P_{m}=1-P_{d}=1-Q\left(\frac{\lambda_{2}-\left(P+\sigma_{n}{ }^{2} / \rho\right)}{\sqrt{2 / N} \cdot\left(P+\sigma_{n}{ }^{2} / \rho\right)}\right)
\end{aligned}
$$

\subsection{Proposed Dynamic Double Threshold Energy Detection (ED) Scheme}

It can be seen from Fig. 3 that performance is degraded sharply as the noise uncertainty coefficient is increasing, which results in severe interferences to the licensed terminals. In order to contend this problem the dynamic threshold scheme is implemented. $\rho^{\prime}$ is the dynamic threshold factor and it is closer to 1 i.e. $\rho^{\prime}>1$ and $\rho^{\prime} \approx 1$. The limits for dynamic double threshold can be defined as [11] [12]:

$$
\left\{\begin{array}{l}
\lambda_{1}{ }^{\prime} \epsilon\left[\lambda_{1} / \rho^{\prime}, \rho^{\prime} \lambda_{1}\right] \\
\lambda_{2}{ }^{\prime} \epsilon\left[\lambda_{2} / \rho^{\prime}, \rho^{\prime} \lambda_{2}\right]
\end{array}\right.
$$

While, considering dynamic-double-threshold under noise uncertainty, (12) and (13) will be modified as:

$$
\begin{aligned}
& P_{d}=\begin{array}{c}
\min \\
\sigma^{2} \epsilon\left[{\sigma_{n}}^{2} / \rho, \rho \sigma_{n}{ }^{2}\right] \lambda_{2}{ }^{\prime} \epsilon\left[\lambda_{2} / \rho^{\prime}, \rho^{\prime} \lambda_{2}\right]
\end{array} \\
& Q\left(\frac{\lambda_{2}{ }^{\prime}-\left(P+\sigma^{2}\right)}{\sqrt{2 / N} \cdot\left(P+\sigma^{2}\right)}\right) \\
& P_{d}=Q\left(\frac{\lambda_{2} / \rho^{\prime}-\left(P+\sigma_{n}^{2} / \rho\right)}{\sqrt{2 / N} \cdot\left(P+\sigma_{n}^{2} / \rho\right)}\right) \\
& P_{f}=\underset{\sigma^{2} \epsilon\left[{\sigma_{n}}^{2} / \rho, \rho \sigma_{n}{ }^{2}\right] \lambda_{2}{ }^{\prime} \epsilon\left[\lambda_{2} / \rho^{\prime}, \rho^{\prime} \lambda_{2}\right]}{\max } \\
& Q\left(\frac{\lambda_{1}^{\prime}-\sigma^{2}}{\sqrt{2 / N} \cdot \sigma^{2}}\right) \\
& P_{f}=Q\left(\frac{\rho^{\prime} \lambda_{1}-\rho \sigma_{n}^{2}}{\sqrt{2 / N} \cdot \rho \sigma_{n}^{2}}\right)
\end{aligned}
$$

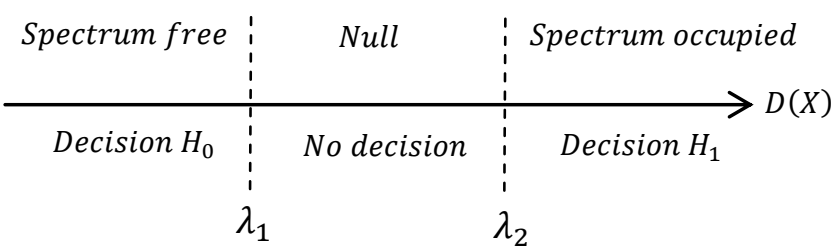

Figure 2. Double threshold based spectrum sensing.

$P_{m}=1-P_{d}=1-Q\left(\frac{\lambda_{2} / \rho^{\prime}-\left(P+\sigma_{n}{ }^{2} / \rho\right)}{\sqrt{2 / N} \cdot\left(P+\sigma_{n}{ }^{2} / \rho\right)}\right)$

\section{SIMULATION RESULTS}

For simulation additive white Gaussian noise (AWGN) channel is considered with signal-to-noise ratio $(\mathrm{SNR})=-15$ $\mathrm{dB}$, and BPSK modulation is used. Number of samples during detection period $(\mathrm{N})$ is 100 , and $P_{f}$ varies from 0 to 1 .

Fig. 3 shows the numerical receiver operating characteristic (ROC) curves for comparison of the single and double threshold scheme for energy detection technique. The curve has been plotted between probabilities of false alarm $\left(P_{f}\right)$ versus probability of detection $\left(P_{d}\right)$. For probability of false alarm $P_{f}$ value at 0.1 the values for probability of detection are $0.4,0.78,0.82$ and 0.88 for single threshold i.e. $\Delta \lambda=0$, and $\Delta \lambda$ values $0.2,0.15,0.1$ respectively. The result shows that at $\Delta \lambda$ equal to 0.1 , performances best among all, it is because as we go on increasing the value of $\Delta \lambda$, the null probability increases and sensing will be performed again. Therefore, appropriate value of $\Delta \lambda$ should be selected for good performance.

From Fig. 4 it is noticed that at probability of false alarm $P_{f}=$ 0.1 , the probability of detection $P_{d}$ values are $0.58,0.4,0.13$, 0.02 and 0 for the noise uncertainty coefficient $\rho$ are $1,1.01$, $1.02,1.03,1.05$ and 1.1 respectively. Thus, we can deduce that with slight increase in $\rho$ there is sharp falloff in $P_{d}$, so it can stated that, very small change in noise variance will severely deteriorate the detection performance. Along these lines, energy detection method is susceptible towards noise uncertainty.

Fig. 5 shows the results of action taken to reduce the problem of noise uncertainty in sensing technique. The graph has been plotted between probability of false alarm $\left(P_{f}\right)$ and probability of detection $\left(P_{d}\right)$ for (16) and (17) in which dynamic double threshold scheme is proposed. From graph, it can be deduce that at $P_{f}=0.1, P_{d}$ values are $0.69,0.66$ and 0.88 for conditions without noise uncertainty, with noise uncertainty and with the proposed dynamic-double-threshold scheme. This indicates that the proposed scheme improves the performance under noise uncertainty condition.

Fig. 6 compares the single threshold and proposed dynamicdouble-threshold scheme under these three conditions:

5.1 With no noise uncertainty.

5.2 With noise uncertainty.

5.3 With noise uncertainty and dynamic threshold.

Here $N U^{*}$ represents noise uncertainty under double threshold scheme, $N U-D^{*}$ represents noise uncertainty under proposed dynamic-double-threshold scheme and $N U$ 
represents noise uncertainty under the single threshold scheme.

From the result, it is concluded that under our proposed scheme, at $P_{f}=0.1, P_{d}$ value is 0.89 and 0.6 for single and proposed scheme considering noise uncertainty. It clearly indicates the performance improvement.

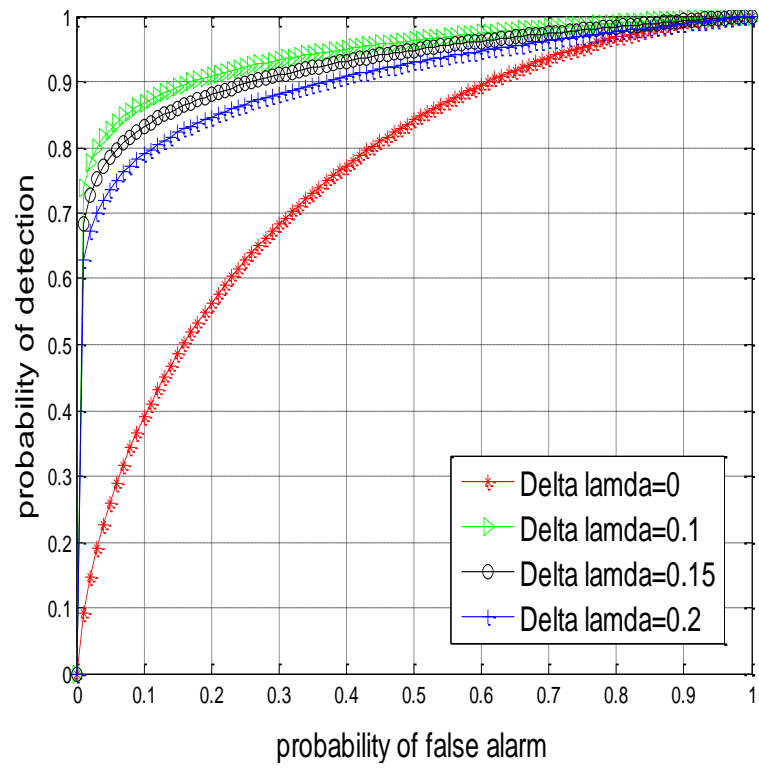

Figure 1. Comparison curve for single and double threshold schemes without noise uncertainty consideration.

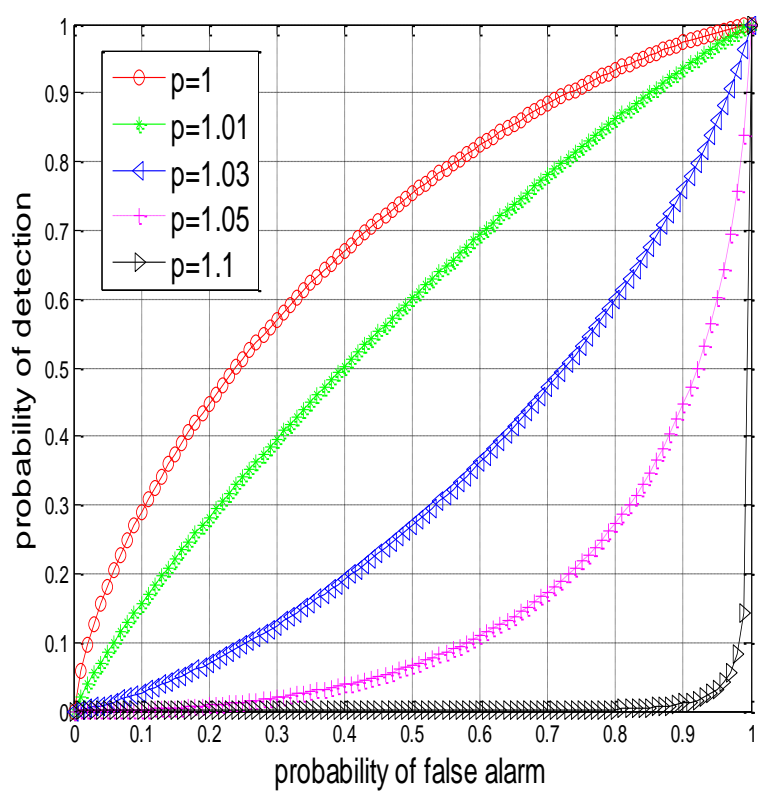

Figure 2. ROC curves of energy detection scheme with different noise uncertainty coefficient $\rho$.

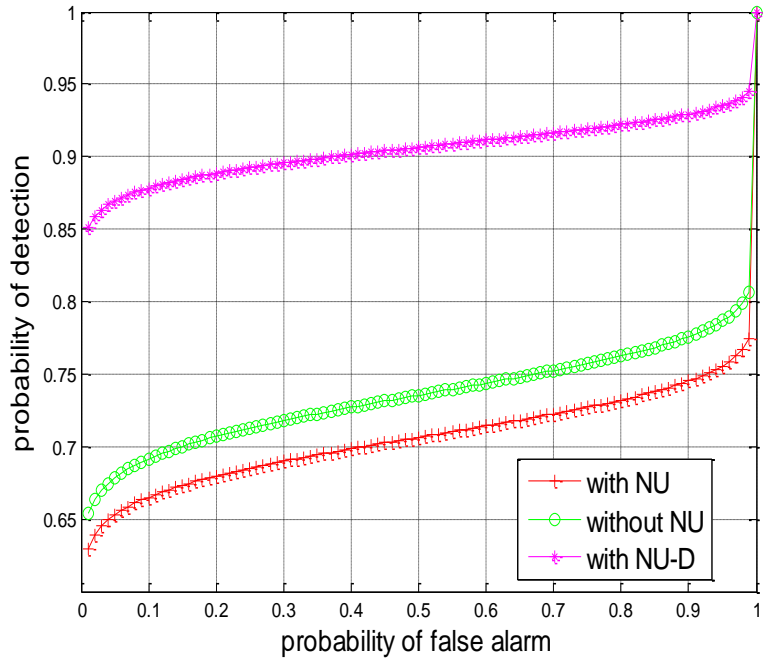

Figure 3. ROC curves of ED scheme with dynamic-double threshold with noise uncertainty.

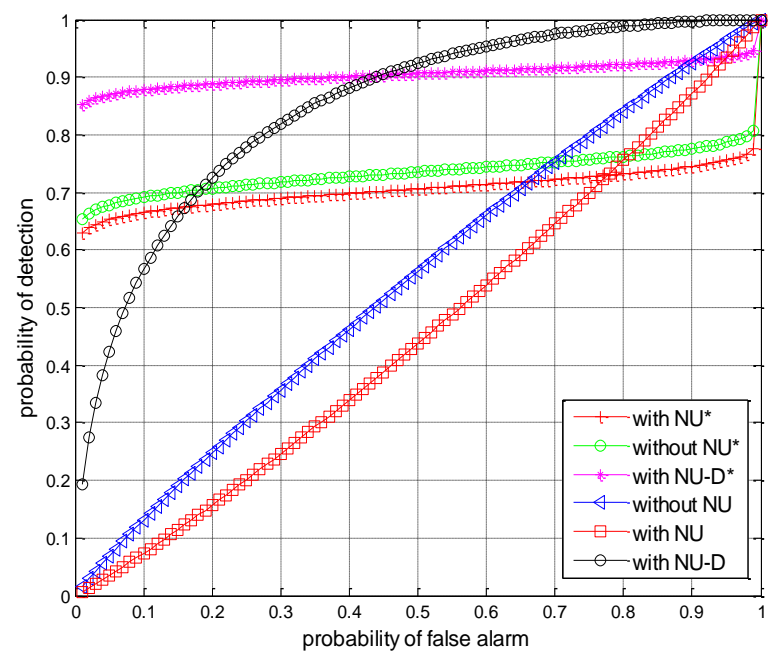

Figure 4. Comparison of ED for single threshold and propopsed scheme with noise uncertainty consideration.

\section{CONCLUSION}

The energy detection technique has been used amongst the basic transmitter detection techniques because of its less intricacy. As this method is very much susceptible towards the noise uncertainty due to continuously changing wireless channel, a novel dynamic-double-threshold spectrum sensing scheme has been proposed in this paper, in order to lessen the effect of varying noise uncertainty. This scheme performs better even under low signal-to-noise ratio (SNR) condition and relentless noise uncertainty. The simulation results show that the proposed technique boosts the performance in terms of detection probability by keeping false alarm probability at a low value. The overall performance of the proposed scheme is based on selection of $\Delta \lambda$ and dynamic threshold coefficient $\rho^{\prime}$. If suitable values for these factors are selected the performance degradation caused by noise uncertainty can be expunged, and radio spectrum utilization can be enhanced. 


\section{ACKNOWLEDGMENTS}

The authors wish to thank their friends and colleagues for the support and encouragement given by them for this work. Finally, authors reserve the most special gratitude to their families. Without their unconditional support and love, this could have been impossible.

\section{REFERENCES}

[1] Federal Communications Commission, "Notice of proposed rulemaking and order: Facilitating opportunities for flexible, efficient, and reliable spectrum use employing cognitive radio technologies," ET Docket No. 03-108, Feb. 2005.

[2] Kolodzy, Paul, and Interference Avoidance. "Spectrum policy task force," Federal Communication Commission, Washington, DC, Rep. ET Docket 02-135, 2002.

[3] Coase, H. Ronald "The federal communications commission," Journal of law and economics 2: pp.1-40, 1959.

[4] S. Haykin, "Cognitive radio: brain-empowered wireless communications," IEEE Journal on Selected Areas in Communications 23(2): $201-220,2005$.

[5] H. Urkowitz, "Energy detection of unknown deterministic signals," Proceedings of the IEEE, vol. 55, no. 4, pp. 523 - 531, April, 1967.

[6] Parikshit Karnik and Sagar Dumbre, "Transmitter Detection Techniques for Spectrum Sensing in CR
Networks," Department of Electrical and Computer Engineering, Georgia Institute of Technology, 2004.

[7] J. Zhu, et al., "Double threshold energy detection of cooperative spectrum sensing in cognitive radio," Proc. 3rd CrownCom, pp.1-5, May, 2008.

[8] Rahul Tandra and Anant Sahai, "SNR Walls for Signal Detection", EEE Journal of Selected Topics in Signal Processing, Vol.2, no.1, pp: 4-17, Feb. 2008.

[9] S. J. Shellhammer, S. Shankar, R. Tandra and J. Tomcik, "Performance of power detector sensors of DTV signals in IEEE 802.22 WRANs," Proc. 1st Int. Workshop on Technology and Policy for Accessing Spectrum, vol. 222, ACM press, New York, NY, Aug. 2006.

[10] S. M. Kay, "Fundamentals of Statistical Signal Processing: Detection Theory," Englewood Cliffs: Prentice-Hall, vol. 2, 1998.

[11] T. L. Jinbo Wu, Guicai and G. Yue, "The performance merit of dynamic threshold energy detection algorithm in cognitive radio systems," The 1st International Conference on Information Science and Engineering (ICISE2009), IEEE Computer Society, 2009.

[12] H. L. Gui-cai Yu, Yu-bin Shao and G. xin Yue, "Dynamic threshold based spectrum detection in cognitive radio systems," Wicom'09 $5^{\text {th }}$ International Conference on Wireless Communications, Networking and Mobile Computing, 2009. 